ARTíCULO CIENTIFICO

\title{
VALORACIÓN NUTRICIONAL DE ENSILAJES DE CORONA DE PIÑA CON ADICION DE HENO Y UREA
}

Michael López-Herrera ${ }^{1}$, Rodolfo WingChing-Jones ${ }^{2}$, Augusto Rojas-Bourrillon ${ }^{2}$

\section{RESUMEN}

El objetivo de esta investigación fue evaluar el efecto de incluir heno como mejorador de la MS y urea como fuente de nitrógeno no proteico (NNP) en la capacidad fermentativa y valor nutricional de ensilajes de coronas de piña como alimento para rumiantes. El trabajo fue realizado utilizando la técnica de micro silos en bolsas de $1 \mathrm{~kg}$. Se establecieron 2 niveles de urea (0, 0,5\% p/p en base fresca), 4 niveles de heno $(0,10,20,30 \% \mathrm{p} / \mathrm{p}$ en base fresca) y un único nivel de inóculo bacterial (1 L/ton) para todos los tratamientos. Conforme se incrementa el contenido de heno en la mezcla ensilada se reduce el $\mathrm{pH}$ obtenido y el contenido de NH3/NT, lo que asegura una adecuada conservación del material, mayores contenidos de heno se asociaron con mayor contenido de MS, que mejora el aporte de nutrimentos, sin embargo, también aumenta los contenidos de FDN, FDA y CEN, lo que podría reducir la capacidad de consumo y la digestibilidad de los ensilados. La inclusión de urea permite mejorar el contenido de PC utilizando nitrógeno no proteico, no obstante conforme se aumenta la inclusión de heno en los ensilados así disminuye el contenido de PC, obteniéndose una reducción entre 4,6 - $34 \%$ en el contenido de PC de los ensilados. Todas las fracciones de la energía disminuyen conforme aumenta el contenido de heno en los tratamientos. De acuerdo al contenido de FDN los tratamientos podrían ser consumidos a razón de 7,99 - 9,17 kg MS día ${ }^{-1}$. La degradabilidad de la PC de los tratamientos fluctuó entre 71 y $88 \% \mathrm{~h}^{-1}$. Se realizaron simulaciones para determinar el potencial productivo de los materiales ensilados de acuerdo al consumo y aporte de PC y de $E N_{L}$, se determinó que los tratamientos ensilados podrían producir entre $0,34-7,65 \mathrm{~kg}_{\text {leche vaca }}^{-1}$ día $^{-1}$.

Palabras clave: Conservación de Forrajes, Nutrición de Rumiantes, Subproductos agrícolas, Aditivos para piña, Ensilaje

\footnotetext{
${ }^{1}$ Universidad de Costa Rica Centro de Investigación en Nutrición Animal. Escuela de Zootecnia. Autor por correspondencia: michael.lopez@ucr.ac.cr

${ }^{2}$ Universidad de Costa Rica Centro de Investigación en Nutrición Animal. Escuela de Zootecnia.

Recibido: 17 de julio 2015

Aceptado: 25 setiembre 2015
} 


\section{ABSTRACT}

Nutritional value of pineapple crown silages with hay and urea: The objective of this study was to evaluate the effect of addition of hay as enhancer MS and urea as a source of non-protein nitrogen (NPN) in the fermentative capacity and nutritional value of pineapple crowns silage as feed for ruminants. It was performed using micro silage method with $1 \mathrm{~kg}$ bags. Established two urea levels (0,0.5\% w / w wet basis), 4 hay levels $(0,10,20,30 \% \mathrm{w} / \mathrm{w}$ wet basis) and a single level of bacterial inoculum $(1 \mathrm{~L} / \mathrm{t})$ for all treatments and one control treatment without additives. With increasing content of hay in the mixture silage $\mathrm{pH}$ and $\mathrm{NH} 3 / \mathrm{NT}$, which ensures an adequate preservation of the material, higher contents of hay were associated with increased Dry Matter (DM) content, which improves the supply of nutrients, however, also increases the contents of NDF, ADF and Ash, which could reduce dry matter intake and digestibility of silage. The inclusion of urea improves the CP content using non-protein nitrogen; however as you increase the inclusion of hay in silage, CP content decreases, resulting in a reduction between 4.6 to $34 \%$ in the CP content of urea included treatments. All energy fractions decrease with increasing the content of hay in treatments, but no differences when using urea. According to NDF, treatments could be consumed at 7.99 to $9.17 \mathrm{~kg} \mathrm{DM}$ day $^{-1}$. The degradability of CP treatments ranged between 71 and $88 \% \mathrm{~h}^{-1}$. Simulations were performed to determine the potential production that silage materials can provide, according to consumption and amount of $P C$ and $N E_{L}$, it was determined that silage treatments could produce between

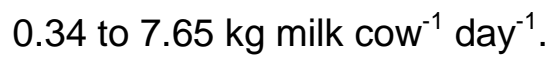

Keywords: Forage preservation, Ruminant Nutrition, Agricultural byproducts, Pineapple additives, Silage 


\section{INTRODUCCIÓN}

El cultivo de la piña genera una cantidad de forraje verde por hectárea que puede ser conservado y utilizado en la alimentación de animales rumiantes; de acuerdo a López et al. (2009) por cada hectárea de cultivo de piña cosechada quedan entre 200 y 250 toneladas de material verde que no tiene uso y que tiene potencial para ser utilizado en la alimentación de rumiantes. También se ha venido utilizando las coronas del fruto como material de alimentación para ganado lechero (Sánchez, 2010), las cuales representan un $9-11 \%$ del peso del fruto $(190-260 \mathrm{~g})$ de acuerdo a la densidad de siembra y cultivar (Rebolledo et al. 2006).

De acuerdo a los trabajos de López et al. (2009), López et al. (2014a) y Rodríguez et al. (2014) con rastrojos de piña y el de Gutiérrez et al. (2003) con cáscara y pulpa de piña, se ha demostrado que estos materiales pueden ser conservados mediante la técnica del ensilaje para ser utilizados en la alimentación de rumiantes. Además señalan que se puede mejorar el contenido de proteína de los ensilados utilizando urea o pollinaza como fuente de nitrógeno (López et al., 2014, Rodríguez et al., 2014). Trabajos realizados en Brasil (Cunha et al., 2009 y Azevedo et al., 2011) respaldan el uso de los subproductos de la industria de la piña, como materiales conservados o utilizados frescos en la alimentación de rumiantes.

López et al. (2014b) señalan que los subproductos de la industria piñera son una buena fuente de fibra de calidad para alimentar a los rumiantes, sin embargo poseen limitaciones en términos de proteína y/o materia seca, lo que limita el aporte de nutrimentos en las formulaciones de animales de mediana y alta producción (Sánchez, 2010). El uso de urea como aditivo, es una práctica común en la elaboración de ensilajes de forrajes con bajo contenido de proteína (Yitbarek y Tamir, 2014); la urea aumenta el contenido de proteína cruda (Neumann et al., 2010) y la estabilidad aeróbica del ensilado (Borges et al., 2011). En Costa Rica Arroyo et al. (2003) reportan beneficios en el uso de la urea, como incremento en el aporte de proteína cuando evaluaron ensilados de pulpa de pejibaye.

Cuando se conservan forrajes con alto contenido de humedad, se incrementa la producción de efluentes y las pérdidas de nutrimentos como consecuencia de estos 
efluentes (Razak et al., 2012); el uso de materiales absorbentes como aditivos para el ensilaje, reduce la producción de efluentes y mejora el contenido de materia seca de los materiales ensilados (Arbabi et al., 2008, Jones y Jones, 1996). Dentro de los aditivos absorbentes más utilizados se encuentran: el heno y rastrojos de gramíneas (McDonald, 1981, Yitbarek y Tamir, 2014), pulpas de cítricos deshidratada (PCD) y fresca, también pulpa de remolacha azucarera y rastrojo de trigo (Arbabi et al., 2008).

El objetivo de esta investigación es evaluar el efecto de utilizar los aditivos, urea y heno de pasto Transvala (Digitaria decumbens, Stent., cv. Transvala) sobre la calidad del proceso fermentativo y calidad nutricional de ensilados de mezclas con coronas de la piña, para aumentar la materia seca y la proteína de este material con potencial forrajero.

\section{MATERIALES Y MÉTODOS}

Las coronas utilizadas en este experimento fueron recolectadas en una finca ubicada en Guápiles, provincia de Limón. La zona se caracteriza por presentar precipitaciones anuales entre $3000-4500 \mathrm{~mm}$ y una temperatura promedio de $25^{\circ} \mathrm{C}$. Las mismas fueron recolectadas al azar de una pila de material dentro de la finca, los materiales estaban frescos ya que habían sido recortados ese mismo día.

Para la conservación del material se utilizaron bolsas de polietileno para empaque al vacío con capacidad para $1 \mathrm{~kg}$ y con un grosor de 0,0063 mm. Cada bolsa se consideró como una unidad experimental. Las coronas recolectadas fueron picadas con cuchillo hasta obtener un tamaño de partícula promedio de $3 \mathrm{~cm}$; a estos se les agregaron de acuerdo a cada tratamiento, 2 niveles de urea $(U)$ (0 y 0,5\% p/p), 4 niveles de heno $(H)$ de pasto Transvala (Digitaria decumbens, Stent., cv. Transvala) $(0,10,20,30 \% \mathrm{p} / \mathrm{p})$ y un único nivel de melaza $(3 \% \mathrm{p} / \mathrm{p})$ e inóculo bacterial artesanal (1 L/ton) para todos los tratamientos. Se preparó una muestra sin aditivos como control. Cada tratamiento contó con 4 repeticiones para un total de 36 micro silos. 
El material fue embolsado para someterlo al proceso de ensilaje; se utilizó una bomba de succión para extraer el aire de las bolsas y favorecer un proceso anaeróbico. Los micro silos se mantuvieron en condiciones ambientales (temperatura de $25 \stackrel{\circ}{\mathrm{C}}$ y humedad relativa $<80 \%$ ) controladas por 60 días.

Al momento de apertura de los micro silos, se realizó una valoración organoléptica de acuerdo a los parámetros indicados por Betancourt et al. (2005). Las mezclas ensiladas fueron llevadas al CINA para ser analizadas. Tanto a las muestras previas como a las muestras ensiladas se les realizaron análisis de materia seca (MS), proteína cruda (PC), extracto etéreo (EE) y cenizas (CEN) de acuerdo a AOAC (1998), carbohidratos no fibrosos (CNF) según Detmann y ValadaresFilho (2010) donde se elimina el efecto de la urea en el cálculo de los CNF. Además fibra detergente neutro (FDN), fibra detergente ácido (FDA) y hemicelulosa (HEM) con la metodología descrita por Van Soest y Robertson (1985). El pH se determinó utilizando un potenciómetro con electrodo de hidrógeno y el nitrógeno amoniacal fue analizado utilizando la metodología descrita por Tobía (2004). Para la estimación del TND se procedió a utilizar las ecuaciones descritas por Weiss (2004) y para la estimación de la energía se utilizaron las ecuaciones de NRC (2001).

Para el análisis de la información se utilizó un modelo de Análisis de Varianza ANOVA de INFOSTAT (2013), de acuerdo a la siguiente ecuación:

$$
y_{i j k}=\mu+H_{i}+U_{j}+(H x U)_{i j}+\varepsilon_{i j k}
$$

Donde:

y = es la variable de respuesta obtenida de la ecuación.

$\mu=$ es la media

$M=$ es el efecto i-esimo del heno en los tratamientos.

$\mathrm{U}=$ es el efecto j-esimo de la urea en los tratamientos.

$\mathrm{MxU}=$ es el efecto de la interacción entre los aditivos urea y heno

$\varepsilon=$ Término de error, donde $\varepsilon \sim N\left(0, \sigma^{2}\right)$

Cuando se determinó significancia de los efectos principales, la comparación entre medias se realizó mediante la prueba de Tukey con un nivel de significancia de 0,05. 


\section{RESULTADOS Y DISCUSIÓN}

A continuación se presenta el efecto de los aditivos utilizados (heno y urea), sobre la calidad del proceso fermentativo y calidad nutricional de los materiales ensilados.

pH: El grado de acidez de los materiales ensilados fue afectado de manera significativa $(p<0,05)$ tanto por la interacción entre la urea y el heno, como por la incorporación de melaza (Cuadro 1), el efecto de la melaza coincide con los resultados descritos por López-Herrera et al. (2009) y el efecto de la urea coincide con los resultados de Rodríguez-Chacón et al. (2014).

Cuadro 1. Medias para las variables del proceso fermentativo y calidad nutricional de las mezclas de coronas de piña con adición de heno y urea, 60 días después del proceso de ensilaje. San José, Costa Rica 2014.

\begin{tabular}{|c|c|c|c|c|c|c|c|c|c|}
\hline & \multicolumn{9}{|c|}{ Tratamientos } \\
\hline & Control & UOHO & U0H10 & UOH2O & UOH30 & U0,5H0 & U0,5H10 & $\mathrm{U} 0,5 \mathrm{H} 20$ & U0,5H30 \\
\hline \multicolumn{10}{|c|}{ Propiedades fermentativas } \\
\hline $\mathrm{pH}$ & $5,2^{\mathrm{c}}$ & $5,9^{c}$ & $4,8^{b}$ & $4,3^{\mathrm{a}}$ & $4,6^{\mathrm{ab}}$ & $7,1^{d}$ & $5,0^{a b}$ & $4,8^{\mathrm{ab}}$ & $4,9^{\mathrm{ab}}$ \\
\hline $\mathrm{NH} 3 / \mathrm{NT}^{*}$ & $10,4^{b}$ & $7,5^{\mathrm{ab}}$ & $3,2^{a}$ & $2,2^{a}$ & $1,7^{\mathrm{a}}$ & $8,1^{\mathrm{ab}}$ & $6,4^{\mathrm{ab}}$ & $3,5^{\mathrm{a}}$ & $2,0^{\mathrm{a}}$ \\
\hline \multicolumn{10}{|c|}{ Composición nutricional (\%MS) } \\
\hline MS (\%) & $6,75^{\mathrm{a}}$ & $7,15^{\mathrm{a}}$ & $13,97^{\mathrm{ab}}$ & $21,37^{\mathrm{bc}}$ & $26,5^{c}$ & $8,11^{\mathrm{a}}$ & $15,0^{\mathrm{ab}}$ & $22,63^{\mathrm{bc}}$ & $31,3^{c}$ \\
\hline PC & $12,15^{b c}$ & $11,90^{b c}$ & $9,95^{\mathrm{ab}}$ & $8,12^{a}$ & $8,04^{a}$ & $12,80^{\mathrm{bc}}$ & $14,38^{\mathrm{C}}$ & $12,53^{b c}$ & $10,27^{\mathrm{ab}}$ \\
\hline EE & $3,15^{b c}$ & $2,20^{a}$ & $3,47^{c}$ & $2,17^{\mathrm{a}}$ & $2,08^{a}$ & $2,35^{\mathrm{ab}}$ & $2,48^{\mathrm{ab}}$ & $2,17^{\mathrm{a}}$ & $2,01^{a}$ \\
\hline Cenizas & $7,25^{a}$ & $8,05^{a}$ & $9,83^{b c}$ & $10,07^{\mathrm{bc}}$ & $10,28^{b c}$ & $8,75^{\mathrm{ab}}$ & $10,03^{b c}$ & $10,30^{b c}$ & $10,85^{c}$ \\
\hline FDN & $54,30^{\mathrm{a}}$ & $53,05^{a}$ & $61,07^{b c}$ & $62,40^{\mathrm{bc}}$ & $66,65^{c d}$ & $60,50^{b}$ & $61,75^{\mathrm{bc}}$ & $64,30^{\mathrm{cd}}$ & $68,10^{d}$ \\
\hline FDA & $26,95^{a}$ & $35,90^{b}$ & $36,70^{b}$ & $39,53^{b c}$ & $45,63^{\mathrm{C}}$ & $37,05^{b}$ & $37,63^{b c}$ & $40,77^{\mathrm{bc}}$ & $45,85^{\mathrm{C}}$ \\
\hline HEM & $27,35^{\mathrm{b}}$ & $24,65^{\mathrm{ab}}$ & $24,37^{\mathrm{ab}}$ & $22,87^{\mathrm{ab}}$ & $21,03^{a}$ & $23,45^{\mathrm{ab}}$ & $24,13^{\mathrm{ab}}$ & $23,53^{\mathrm{ab}}$ & $22,25^{\mathrm{ab}}$ \\
\hline $\mathrm{CNF}^{\star}$ & $23,15^{\mathrm{cd}}$ & $24,80^{d}$ & $15,37^{\mathrm{ab}}$ & $17,24^{b c}$ & $12,96^{\mathrm{a}}$ & $21,21^{\mathrm{cd}}$ & $16,98^{\mathrm{ab}}$ & $16,32^{\mathrm{ab}}$ & $14,28^{\mathrm{a}}$ \\
\hline
\end{tabular}


Los tratamientos sin heno fueron los que presentaron valores mayores de $\mathrm{pH}$, pues conforme se aumentó la cantidad de heno en la mezcla, se redujo el $\mathrm{pH}$ final de cada tratamiento ensilado. Lo que concuerda con lo reportado por Hiriart (2008) y McDonald (1981) quienes afirman que los ensilados de forrajes con altos contenidos de humedad presentan un $\mathrm{pH}$ más elevado, que los ensilados de forrajes que poseen menos de $70 \%$ de humedad. El agregar aditivos altos en nitrógeno también afectó el valor $\mathrm{pH}(\mathrm{p}<0,05)$, este valor fue más alto al agregar urea a la mezcla, estos resultados coinciden con los comportamientos encontrados por Borges et al. (2011) quienes trabajaron con urea y Gutiérrez et al. (2003) quienes agregaron pollinaza a los ensilados, quienes obtuvieron mayores valores de pH conforme se incrementó la inclusión de fuentes altas en nitrógeno.

Valores de $\mathrm{pH}$ en los materiales ensilados fueron mayores a los obtenidos por Betancourt (2004) $(3,55)$ y Cubero $(2010)(3,47$ - 3,67) en ensilados de maíz, también es mayor que los valores descritos por López-Herrera et al. (2009) en mezclas ensiladas de rastrojos de piña con niveles crecientes de PCD y también mayores a los datos generados en la investigación de Cunha et al. (2009) $(3,4-3,5)$ con ensilados de cáscara y pulpa.

Nitrógeno amoniacal (NH3/NT): tanto la inclusión de melaza como la incorporación de heno afectaron de manera significativa $(p<0,05)$ el contenido de nitrógeno amoniacal de las mezclas ensiladas, no así la inclusión de urea (Cuadro 1). De esta manera, los tratamientos a los que se les agregó melaza redujeron la concentración de NH3/NT en casi dos puntos porcentuales, sin embargo al adicionar el heno la diferencia con respecto al tratamiento control fue de 4 - 8,7 puntos porcentuales, aunque no hubo diferencia conforme se agregó más heno.

A pesar de los valores de $\mathrm{pH}$ obtenidos (Cuadro 1), todos los tratamientos con heno mostraron valores menores de $7 \%$ de $\mathrm{NH} 3 / \mathrm{NT}$, lo que indica que han sido conservados mediante un buen proceso de ensilaje, de acuerdo a lo publicado por Betancourt et al. (2005). Los tratamientos sin adición de heno presentaron mayores concentraciones de $\mathrm{NH} 3 / \mathrm{NT}$, lo que es indicativo de malos procesos de fermentación y actividad de bacterias clostridiales que descomponen las proteínas de los forrajes, debido a altas concentraciones de humedad (Moore y Peterson, 1995, McDonald, 1981). Aunque se ha encontrado que al aumentar la inclusión de urea en las mezclas para ensilaje, aumenta la concentración de N-NH3/NT (Borges et al., 2011, Rodríguez-Chacón et al., 2014 y LópezHerrera et al., 2014a); en este experimento no fue posible determinar diferencias entre los tratamientos por inclusión de urea. 
Todos los tratamientos con adición de heno mostraron concentraciones de N-NH3/NT menores a las publicadas en las investigaciones de Gutiérrez et al. (2003) (13,38 23,17\% N-NH3/NT), para ensilados de cáscaras y pulpa de piña mezcladas con pollinaza; de Rodríguez-Chacón et al. (2014) (2,61 - 7,10\% N-NH3/NT) con rastrojos de piña, urea y melaza deshidratada con minerales (MDM) en los tratamientos con niveles de inclusión de urea de $1 \%$. Además fueron menores que los valores reportados por Cubero et al. (2010) $(6,01$ - 8,23\% N-NH3/NT) en ensilados de maíz, aunque similares a los valores encontrados por Castillo et al. (2009) (2,45 - 3,92\% N-NH3/NT) con ensilados de combinaciones de maíz y vigna (Vigna radiata). Todos los tratamientos sin inclusión de heno presentaron valores mayores a los citados por los autores anteriores, salvo los citados en el trabajo de Gutiérrez et al. (2003).

Materia seca: El contenido de MS de los tratamientos se ve afectado de manera significativa $(p<0,05)$ por la inclusión de heno; la inclusión de urea no afecta de ninguna manera esta variable (Cuadro 1). El contenido de MS de las coronas sin aditivos es bajo debido a estructuras en las hojas de la planta y las coronas donde se almacena el agua (Bartholomew et al., 2003), por lo que conforme aumenta la inclusión de heno, se incrementa el valor de esta fracción en la mezcla ensilada, debido a la concentración de esta fracción en el heno (80,22 $44,82 \%$ MS) (WingChing y Alvarado, 2009).

Conforme se incrementa el contenido de MS de la mezcla ensilada, se mejoran los parámetros fermentativos de la mezcla ensilada, tal y como se observa en el Cuadro 1, esto coincide con lo publicado en los trabajo de McDonald (1981), Moore y Peterson (1995) e Hiriart (2008). El mismo efecto se encontró en los trabajos de López-Herrera et al. (2009) y Gutiérrez et al. (2003), quienes trabajaron con materiales altos en materia seca como la PCD y la pollinaza, respectivamente. El contenido de MS de los tratamientos con adición de heno, es mayor o similar a los valores obtenidos por Lallo et al. (2003) (14,78\% MS) con ensilados de subproductos de fruto de piña; Azevedo et al. (2011) (13,91\% MS) con cáscaras y pulpa de piña; Rodríguez-Chacón et al. (2014) (9,2 $11,5 \%$ MS) con ensilados de rastrojos de piña, urea y MDM; finalmente similares a los reportados por Cunha et al. (2009) (12,3 - 13,4\% MS) con ensilados de cáscaras y pulpa de piña. Por otra parte, los tratamientos a los que no se les agregó heno, presentaron 
valores menores a los reportados por los autores anteriores, aunque similares a los obtenidos por López-Herrera et al. (2014a).

Al utilizar las tablas de requerimientos del NRC (2001) una vaca de $454 \mathrm{~kg}$ de peso corporal en lactancia media, que produce 10 kilogramos de leche con $4 \%$ grasa/día, debe consumir 12,4 kg MS/día con una dieta menor al 68\% de TND. De acuerdo a la ecuación propuesta por Belyea et al. (1996), la que relaciona la FDN con consumo de MS, los tratamientos evaluados fluctúan entre 1,76 - 2,02\% del peso corporal, es decir que los ensilados podrían proveer entre 7,99 - 9,17 kg de MS correspondientemente, con esta predicción se puede reafirmar el hecho de que las mezclas ensiladas evaluadas no pueden ser utilizadas como único alimento en una dietas de vacas en producción, debido a que no llenan los requerimientos de MS, por lo que deben ser utilizadas como parte de una ración total (Cuadro 2).

Cuadro 2. Cantidad de MS y material verde (MV) (kg/día) estimada a partir del contenido de FDN en los tratamientos evaluados para alimentar una vaca de $454 \mathrm{~kg}$ de peso corporal. San José, Costa Rica 2014.

\begin{tabular}{lcc}
\hline & Kg MS/día & Kg MV/día \\
\hline Control & 9,17 & 134,9 \\
U0H10 & 8,98 & 124,7 \\
U0H20 & 8,9 & 63,6 \\
U0H30 & 8,71 & 40,7 \\
U0,5H0 & 8,17 & 30,83 \\
U0,5H10 & 8,98 & 110,9 \\
U0,5H20 & 8,81 & 58,7 \\
U0,5H30 & 8,49 & 37,6 \\
\hline FDN (Fibra Detergente Neutro), U (Urea), H (Heno) &
\end{tabular}

Proteína cruda: El contenido de proteína de los materiales ensilados se ve afectado tanto por la inclusión de urea, como por la inclusión de heno en la mezcla $(p<0,05)$. La PC 
aumenta de forma directamente proporcional a la inclusión de urea, tal y como ocurre en los trabajos de Gutiérrez et al. (2003), Rodríguez-Chacón et al. (2014) y López-Herrera et al. (2014a), quienes agregaron pollinaza o urea en ensilajes de subproductos de piña. Sin embargo, al incluir heno en la mezcla ensilada, el contenido de PC disminuye, debido al bajo contenido de esta fracción presente en el heno $(3,42 \pm 0,11 \%$ MS) (WingChing y Alvarado, 2009). Las diferencias en el contenido de PC entre los tratamientos sin heno y los tratamientos con adición de heno, fue de hasta 4,6 puntos porcentuales en los tratamientos sin adición urea y de hasta 2,01 puntos porcentuales en los tratamientos con adición de urea (Cuadro 3).

Todos los ensilados evaluados, poseen un contenido de PC mayor o similar que los valores reportados por: López et al. (2009) (7,08 - 8,24\% MS), Lallo et al. (2003) (8,83\% MS), Cunha et al. (2009) (7,30 - 7,80\% MS), Azevedo et al. (2009) (7,09\% MS) y Correia et al. (2006) (7,37\% MS), debido a que las coronas poseen un mayor contenido de PC que otros subproductos de la piña (López et al. 2014b).

Los tratamientos con inclusión de urea, presentaron concentraciones de PC menores o similares, a los obtenidos por Rodríguez-Chacón et al. (2014) (8,40 - 18,30\% MS) con ensilados de rastrojos de piña, urea y MDM; Elizondo-Salazar y Campos-Granados (2014) $(17,25$ - 20,06\% MS) con ensilados de cáscaras de piña con heno y urea y López et al. (2014a) $(8,17$ - 30,07\% MS) con ensilados de rastrojos de piña con urea. Este valor menor en los tratamientos con urea puede deberse al efecto del heno en la mezcla.

Al utilizar las ecuaciones establecidas por Yan y Agnew (2004) para estimar la tasa de degradación de la proteína de los tratamientos, se observa que la misma fluctúa entre $71,4-88,3 \%$ de acuerdo a como se describe en el Cuadro 3. Estos autores señalan que la degradabilidad de la proteína a una tasa de pasaje de 0,02/h (consumo 1x) en un ensilaje aumenta conforme la concentración de proteína en el material aumenta, tal y como ocurre con estos materiales. La tasa de degradación estimada de la proteína es influenciada por la interacción entre la urea y el heno, de esta manera la urea aumenta la concentración de PC, lo que aumenta la tasa de degradación, sin embargo el heno reduce la concentración de PC y reduce así la tasa de degradación estimada, generando los resultados mostrados en el Cuadro 3. 
Al realizar una simulación del potencial de producción que tienen los materiales obtenidos en este trabajo, se puede observar (Figura 1) que al utilizar como referencia la investigación de Sánchez (2010), quien indica que es posible suministrar hasta $15 \mathrm{~kg}$ de corona fresca en la alimentación de vacas lecheras, sin afectar de manera negativa su producción. De acuerdo al contenido de PC y al consumo de cada tratamiento, el potencial de producción de los ensilados es de entre 1,06 y 4,01kg lechevaca-1 día-1, cuando el animal consume 15 kg MVvaca-1 día-1 y no más de 1,34kg lechevaca-1 día-1, cuando el consumo es de $5 \mathrm{~kg}$ MVvaca-1 día-1.

Cuadro 3. Estimación de la degradabilidad de la proteína y la materia seca de acuerdo a la tasa de pasaje, de los ensilados de rastrojos de piña con inclusión de urea (\% $\mathrm{p} / \mathrm{p}$ en base fresca) y heno (\% p/p en base fresca), con 60 días de proceso de ensilaje. San José, Costa Rica 2014.

\begin{tabular}{l|ll}
\hline \multicolumn{2}{l}{} & \multicolumn{2}{l}{ Degradabilidad de la PC } \\
\hline Tasa de pasaje $\% \mathbf{h}^{-1}$ & $\mathbf{0 , 0 2}$ & $\mathbf{0 , 0 8}$ \\
\hline Tratamientos & $85^{\mathrm{bc}}$ & $78^{\mathrm{bc}}$ \\
Control & $83^{\mathrm{ab}}$ & $76^{\mathrm{bc}}$ \\
$0 \%$ urea y $0 \%$ heno & $81^{\mathrm{ab}}$ & $75^{\mathrm{ab}}$ \\
$0 \%$ urea y $10 \%$ heno & $78^{\mathrm{a}}$ & $72^{\mathrm{a}}$ \\
$0 \%$ urea y $20 \%$ heno & $78^{\mathrm{a}}$ & $71^{\mathrm{a}}$ \\
$0 \%$ urea y $30 \%$ heno & $88^{\mathrm{c}}$ & $81^{\mathrm{c}}$ \\
$0,5 \%$ urea y $0 \%$ heno & $88^{\mathrm{c}}$ & $80^{\mathrm{c}}$ \\
$0,5 \%$ urea y $10 \%$ heno & $85^{\mathrm{bc}}$ & $78^{\mathrm{bc}}$ \\
$0,5 \%$ urea y $20 \%$ heno & $82^{\mathrm{ab}}$ & $75^{\mathrm{ab}}$ \\
$0,5 \%$ urea y 30\% heno & & \\
\hline a,b Valores en la misma columna con diferentes letras son estadísticamente \\
diferentes (p<0,05)
\end{tabular}




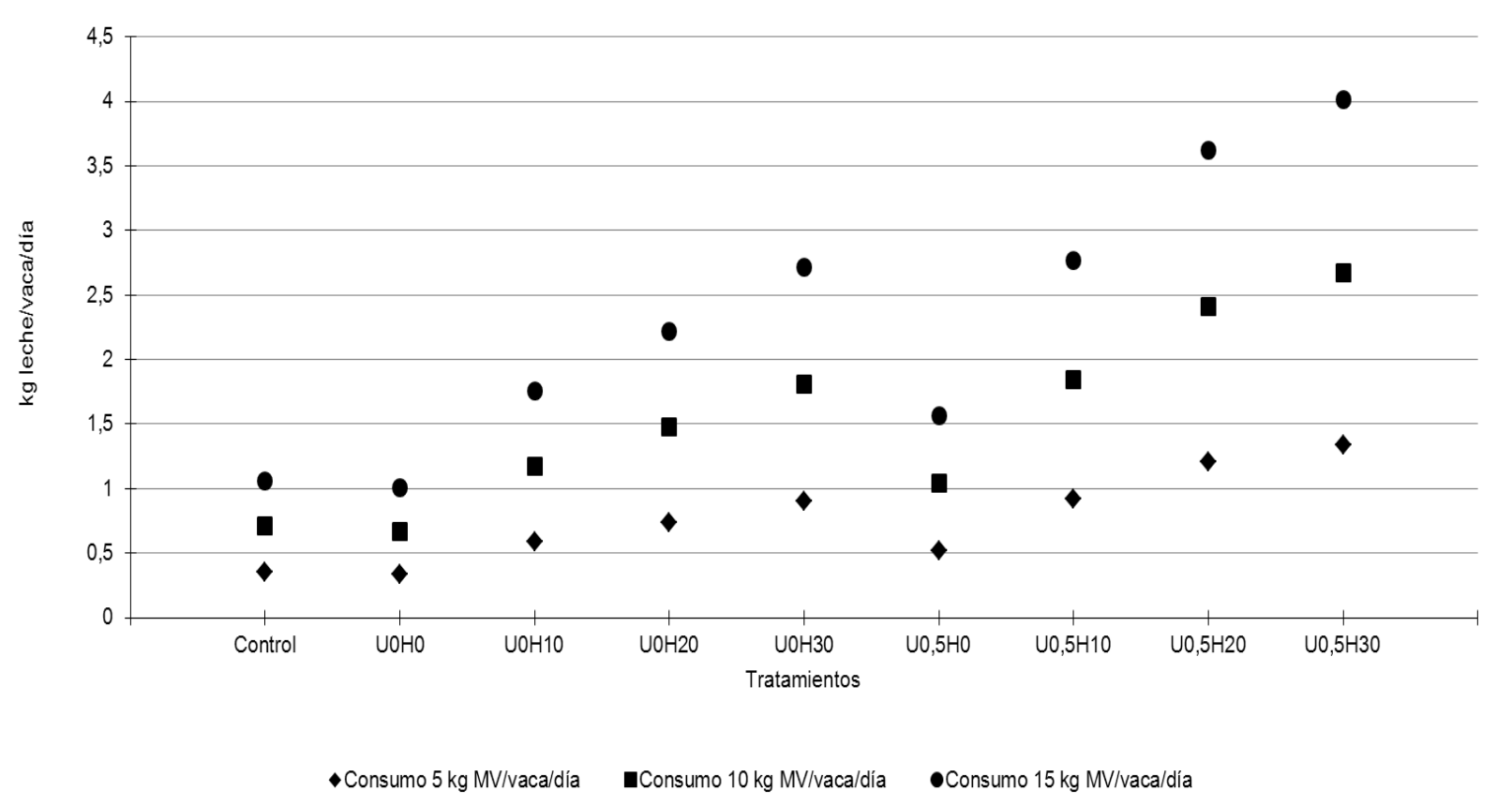

Figura 1. Potencial de producción por gramos de proteína cruda, de los ensilados de corona de piña con pulpa de cítricos deshidratada, heno y urea de acuerdo al nivel de consumo de Materia verde (MV) (5, 10 y $15 \mathrm{~kg} \mathrm{MV/día).} \mathrm{H=(Heno)} \mathrm{y}$ $\mathrm{U}=($ Urea $)$

Las diferencias entre los tratamientos, se debe tanto a la adición de heno y como a la incorporación de urea en la mezcla ensilada, ya que el heno aumenta la cantidad de materia seca consumida por kilogramo de material verde; por su parte la urea aumenta la cantidad de proteína degradable en el rumen; al ser fuente de nitrógeno no proteico (NNP) (Yitbarek y Tamir 2014).

Fibra en detergente neutro: La concentración de FDN se vio influenciada por la inclusión de heno en las mezclas ensiladas $(p<0,05)$. La adición de heno aumenta la concentración de FDN, con respecto a los tratamientos sin heno, debido al contenido de FDN en el heno (66,14\% MS) (WingChing y Alvarado 2009), aunque no se encontró diferencias entre los tratamientos con inclusión de heno (Cuadro 1). De acuerdo a Sánchez y Soto (1998) el 
aumento en el contenido de FDN podría afectar la capacidad de consumo de estos materiales por parte de los animales, y esto refuerza lo publicado por Van Soest y Robertson (1985) acerca del efecto de la FDN sobre el consumo de materia seca de los rumiantes.

El contenido de FDN de los tratamientos con inclusión de heno, fue mayor o similar que los valores obtenidos por Elizondo-Salazar y Campos-Granados (2014) (50,49 - 57,12\% MS) con ensilados de cáscaras de piña con heno y urea; López-Herrera et al. (2009) (41,80 - 56,00 \% MS) con ensilados de rastrojos de piña y PCD; López-Herrera et al. (2014a) $(54,82$ - 65,12\% MS) con ensilados de rastrojos de piña y urea; Castillo et al. (2009) $(50,10$ - 61,26\% MS) con ensilados de maíz y vigna (Vigna radiata); Cubero et al. (2010) $(58,64$ - 66,48\% MS) con ensilados de maíz con diferentes tipos de inóculos bacterianos.

Como se mencionó anteriormente al utilizar la ecuación propuesta por Belyea et al. (1996), que relaciona la FDN con consumo de MS, los tratamientos evaluados permitirían al rumiante, consumir $1,76-2,02 \%$ del peso corporal. Al comparar estos valores con los que podrían proveer pastos tropicales como: el pasto Estrella africana (Cynodon nlemfuensis) (1,86 \%) (Villalobos y Arce, 2014); el pasto Ratana (Ischaemumindicum) $(1,81 \%)$ (Boschini-Figueroa et al., 2014) y el pasto King grass (Pennisetm purpureum) $(1,59 \%)$ (Chacón-Hernández y Vargas-Rodríguez, 2009); se puede observar que los ensilados evaluados son de similar o mejor calidad que los forrajes anteriormente mencionados.

Fibra en detergente ácido: Esta variable fue influenciada de manera significativa $(p<0,05)$ por la adición de heno en la mezcla ensilada. Al igual que con la FDN, la concentración de FDA aumenta conforme se incrementa la inclusión de heno (Cuadro 3),

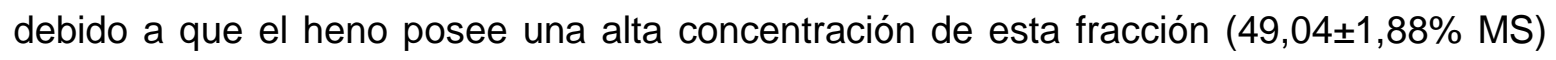
(WingChing y Alvarado 2009). Esto supondría que, los ensilados donde se hizo mayor inclusión de heno son menos digestibles que los materiales con menor inclusión de este aditivo, lo que concuerda con lo publicado por Van Soest et al. (1991) y Sánchez y Soto (1998), quienes indican que niveles altos de FDA se relacionan con bajos niveles de digestibilidad en forrajes.

El contenido de FDA en los tratamientos evaluados fue mayor o similar que los valores obtenidos por Elizondo-Salazar y Campos-Granados (2014) (31,86 - 37,12\% MS) con 
ensilados de cáscaras de piña con heno y urea; López-Herrera et al. (2009) (23,60 $32,20 \%$ MS) con ensilados de rastrojos de piña y PCD; Gutiérrez et al. (2003) (35,39 $36,93 \%$ MS) con ensilados de cáscaras y pulpa de piña y pollinaza; Rogério et al. (2007) $(34,41 \%$ MS) con planta entera de piña deshidratada; Cunha et al. (2009) $(31,91-32,6 \%$ MS) con ensilados de cáscara y pulpa de piña; Cubero et al. (2010) (37,00 - 40,30\% MS) con ensilados de maíz y Castillo et al. (2009) (28,52 - 36,52\% MS) con ensilados de maíz con vigna (Vigna radiata).

Hemicelulosa (HEM): Los contenidos de Hemicelulosa fueron influenciados por el contenido de heno $(p<0,05)$, disminuyendo el contenido de HEM conforme se aumenta la inclusión de heno. El tratamiento control fue el que mayor contenido de HEM presentó, por otra parte, el tratamiento con menor contenido de HEM fue el de mayor inclusión de heno pero sin inclusión de urea (Cuadro 1).

Los valores obtenidos en esta investigación son mayores o similares a los publicados por López-Herrera et al. (2014a) (19,95 - 25,67\% MS) con ensilados de rastrojos de piña con urea, Elizondo-Salazar y Campos-Granados (2014) (15,89 - 19,99\% MS) con ensilados de cáscaras de piña con heno y urea y Castillo et al. (2009) $(21,98-24,74 \%$ MS) con ensilados de maíz y vigna (Vigna radiata). Aunque menores a los obtenidos por Cubero et al. (2007) (26,18 - 31,64\% MS) con ensilados de maíz. De acuerdo a Weiss et al. (1989), forrajes con mayores contenidos de hemicelulosa, son más digestibles ya que esta parte de la fibra es aprovechada más rápidamente por lo rumiantes.

Carbohidratos no fibrosos: Al utilizar la ecuación de corrección del trabajo de Detmann y Valadares-Filho (2010), se determinó que esta fracción se ve influenciada por la inclusión de heno en las mezclas $(p<0,05)$, de esta manera, se encontró que al agregar heno a la mezcla se reduce la concentración de los CNF, sin embargo no se encontraron diferencias entre los tratamientos al agregar más heno a la mezcla (Cuadro 1).

El contenido de CNF en todos los tratamientos fue menor al publicado por Azevedo et al. (2011) $(27,20 \%$ MS) con subproductos de las plantaciones de piña y López-Herrera et al. (2009) (23,50 - 39,10\% MS) con ensilados de rastrojos de piña con PCD. Aunque mayores a los datos publicados por Rodríguez-Chacón et al. (2014) (8,87 - 11,4\% MS). 
Los tratamientos con heno mostraron concentraciones similares a las de los trabajos de López-Herrera et al. (2014a) (16,44 - 17,70\% MS) con ensilados de rastrojos de piña con urea y Rogério et al. (2007) con plantas enteras de piña deshidratadas (14,07\% MS), pero los tratamientos sin heno tuvieron concentraciones mayores, lo que se traduce en una mayor digestibilidad del forraje (Mertens, 1996).

Cenizas (CEN): En cuanto a la fracción mineral, sólo la adición de heno genera diferencias entre los tratamientos $(p<0,05)$. Se encontró que al agregar heno a la mezcla se aumenta la concentración de los CEN, sin embargo no se encontraron diferencias entre los tratamientos al agregar más heno a la mezcla (Cuadro 1).

Los valores obtenidos en los tratamientos sin heno son menores a los valores publicados por Lallo et al. (2003) (17,02\% MS) con cáscaras de piña; Pereira et al. (2009) (10,70\% MS) con cáscaras y pulpa de piña; Rogério et al. (2007) (9,20\% MS) con plantas enteras de piña deshidratadas y Rodríguez-Chacón et al. (2014) $(9,10$ - 13,6\% MS) con ensilados de rastrojos de piña, urea y MDM. Los tratamientos con inclusión de heno presentaron valores similares a los valores reportados por los autores anteriores y a los obtenidos por Correia et al. (2006) (10,08\% MS) con cáscaras y pulpa de piña, aunque mayores a los publicados por Cunha et al. (2009) (4,80 - 6,05\% MS) con ensilados de cáscaras y pulpa de piña y Elizondo-Salazar y Campos-Granados (2014) (15,89 - 19,99\% MS) con ensilados de cáscaras de piña con heno y urea, debido a diferencias en el contenido de nutrimentos en los diferentes subproductos de la planta de piña (López-Herrera et al. $2014 b)$ y al contenido de cenizas en el heno $(12,09 \pm 0,75 \%$ MS) (WingChing y Alvarado 2009).

\section{Caracterización energética de los tratamientos ensilados}

TND: Los tratamientos evaluados fueron afectados de forma negativa por el contenido de heno $(p<0,05)$, disminuyendo el aporte energético de los ensilados conforme se aumenta el contenido de heno en la mezcla (Cuadro 4). Se encontró que la diferencia entre el tratamiento control y los tratamientos con $30 \%$ de heno fue $9,0-9,15$ puntos porcentuales menor en los tratamientos con heno; esta diferencia es debida al menor contenido de TND presente en el heno de pasto Transvala (Digitaria decumbens) $(46,40 \%$ TND) (WingChing 
y Alvarado, 2009).No se encontraron diferencias entre los tratamientos con niveles intermedios de heno.

El contenido de TND de todos los tratamientos es mayor que los valores reportados por Rodríguez-Chacón et al. (2014) (52,70 - 52,80\% TND) con ensilados de rastrojos de piña, urea y MDM y Rogério et al. (2007) (55,95\% TND) con plantas enteras de piña deshidratadas.

Cuadro 4. Medias para las variables de la densidad energética de las mezclas de coronas de piña con adición de heno y urea, 60 días después del proceso de ensilaje. San José, Costa Rica 2014.

\begin{tabular}{|c|c|c|c|c|c|c|c|c|c|}
\hline & \multicolumn{9}{|c|}{ Tratamientos } \\
\hline & Control $^{*}$ & ЧOHO & U0H10 & UOH2O & UOH30 & U0,5HO & U0,5H10 & U0,5H2O & U0,5H3O \\
\hline \multicolumn{10}{|l|}{ Perfil energético* } \\
\hline TND (\%) & $65,80^{\mathrm{b}}$ & $63,20^{\mathrm{ab}}$ & $62,67^{\mathrm{ab}}$ & $60,80^{\mathrm{ab}}$ & $56,80^{\mathrm{a}}$ & $62,40^{\mathrm{ab}}$ & $62,06^{\mathrm{ab}}$ & $59,90^{\mathrm{ab}}$ & $56,65^{a}$ \\
\hline ED (Mcal/kg) & $2,90^{\mathrm{C}}$ & $2,79^{\mathrm{ab}}$ & $2,76^{\mathrm{ab}}$ & $2,68^{a b}$ & $2,50^{\mathrm{a}}$ & $2,75^{\mathrm{ab}}$ & $2,74^{\mathrm{ab}}$ & $2,64^{\mathrm{ab}}$ & $2,50^{\mathrm{a}}$ \\
\hline EM (Mcal/kg) & $2,38^{c}$ & $2,28^{\mathrm{ab}}$ & $2,27^{\mathrm{ab}}$ & $2,20^{a b}$ & $2,05^{\mathrm{a}}$ & $2,26^{\mathrm{ab}}$ & $2,24^{\mathrm{ab}}$ & $2,17^{\mathrm{ab}}$ & $2,05^{a}$ \\
\hline $\mathrm{EN}_{\mathrm{L}}(\mathrm{Mcal} / \mathrm{kg})$ & $1,49^{c}$ & $1,43^{\mathrm{ab}}$ & $1,42^{\mathrm{ab}}$ & $1,37^{\mathrm{ab}}$ & $1,28^{a}$ & $1,41^{\mathrm{ab}}$ & $1,40^{\mathrm{ab}}$ & $1,35^{\mathrm{ab}}$ & $1,27^{\mathrm{a}}$ \\
\hline $\mathrm{EN}_{\mathrm{L}}(\mathrm{Mcal} / \mathrm{kg})_{(3 \mathrm{X})}$ & $1,42^{c}$ & $1,38^{\mathrm{ab}}$ & $1,37^{\mathrm{ab}}$ & $1,34^{\mathrm{ab}}$ & $1,28^{\mathrm{a}}$ & $1,37^{\mathrm{ab}}$ & $1,36^{\mathrm{ab}}$ & $1,33^{\mathrm{ab}}$ & $1,27^{\mathrm{a}}$ \\
\hline $\mathrm{EN}_{\mathrm{M}}(\mathrm{Mcal} / \mathrm{kg})$ & $1,50^{c}$ & $1,42^{\mathrm{ab}}$ & $1,40^{\mathrm{ab}}$ & $1,34^{\mathrm{ab}}$ & $1,20^{\mathrm{a}}$ & $1,39^{\mathrm{ab}}$ & $1,38^{\mathrm{ab}}$ & $1,31^{a b}$ & $1,20^{a}$ \\
\hline $\mathrm{EN}_{\mathrm{G}}(\mathrm{Mcal} / \mathrm{kg})$ & $0,91^{\mathrm{c}}$ & $0,83^{\mathrm{ab}}$ & $0,82^{\mathrm{ab}}$ & $0,76^{\mathrm{ab}}$ & $0,64^{a}$ & $0,81^{\mathrm{ab}}$ & $0,80^{\mathrm{ab}}$ & $0,73^{\mathrm{ab}}$ & $0,63^{a}$ \\
\hline
\end{tabular}

$\mathrm{U}$ (urea), $\mathrm{H}$ (Heno), Nutrimentos digestibles totales (TND), energía digestible (ED), energía metabolizable $(E M)$, energía neta de lactación $\left(E N_{L}\right)$, energía neta de mantenimiento $\left(E N_{M}\right)$, energía neta de ganancia de peso $\left(\mathrm{EN}_{\mathrm{G}}\right)$

*Energías calculadas en base materia seca

${ }^{a, b}$ Valores en la misma fila con diferentes letras son estadísticamente diferentes $(p<0,05)$

Los tratamientos por debajo de $20 \%$ de inclusión de heno son mayores o similares a los valores reportados en las investigaciones de López-Herrera et al. (2014a) (60,6 - 63,8\% TND) con ensilados de rastrojos de piña y urea; López-Herrera et al. (2009) $(59,03-$ $67,14 \%$ TND) con ensilados de rastrojos de piña con PCD; Correia et al. (2006) $(57,81 \%$ TND) y Pereira et al. (2009) (57,17\% TND) con cáscaras y pulpa de piña. También son similares a los valores publicados en los trabajos de Cubero et al. (2010) $(57,65-66,80 \%$ TND) y Betancourt (2004) (60,91\% TND) con ensilados de maíz; Castillo et al. (2009) 
(63,87\% TND) con ensilados de maíz con vigna (Vigna radiata) y Villalobos y Arce (2014) $(59,45-63,07 \%$ TND) con pasto Estrella africana.

Con base en lo descrito en el párrafo anterior, se podría decir que el contenido energético de los ensilados evaluados es comparable con el de forrajes de buena calidad, debido a que los subproductos del cultivo de la piña poseen un buen contenido energético $(59,2$ $66,6 \%$ TND), sin embargo su bajo contenido de proteína cruda (3,5 - 10,1\% MS), FDN $(53,2-70,6 \%$ MS) y FDA $(23,7$ - 60,6\% MS) puede limitar el consumo y aprovechamiento por parte del animal (López-Herrera et al., 2014b).

Energía neta de lactancia $\left(E N_{L}\right)$. El material con mayor contenido de energía para la producción de leche, fue el tratamiento control, reduciéndose el contenido energético conforme se eleva el contenido de heno en la mezcla $(p<0,05)$ (Cuadro 4). Los valores están expresados en una vez mantenimiento (1X), al expresar la energía en tres veces mantenimiento $(3 \mathrm{X})$, los valores de energía se reducen, debido a una disminución en la digestibilidad de los alimentos al aumentar la tasa de consumo (Eastridge, 2002) (Cuadro 4).

De acuerdo a las tablas de requerimientos del NRC (2001) una vaca de $454 \mathrm{~kg}$ de peso corporal, en media lactación que produce 10, 20 y 30 kilogramos de leche con 4\% grasa y $3,5 \%$ de proteína, debe consumir 12,4; 16,0 y 19,5 kg MS/día respectivamente, la cantidad de energía que provee cada uno de los niveles de consumo debe ser 15,6; 23,2 y 30,9 Mcal ENL/día, para que el animal pueda sostener el correspondiente nivel de producción.

En la Figura 2 es posible observar que conforme aumenta el consumo de ensilado, aumenta la cantidad de leche producida, esto es esperable debido a que hay un mayor aporte de nutrimentos al animal para aumentar la producción. También se puede observar una tendencia al aumento del potencial productivo de los tratamientos con heno, debido a que este aditivo permite una mayor cantidad de materia seca consumida y a la vez una mayor cantidad de energía consumida que puede ir para la producción de leche. 
Todos los tratamientos con aditivos, tienen más potencial para la producción de leche con respecto al tratamiento control. Los materiales ensilados permiten una producción de 0,64 - 2,65 kg de lechevaca-1 día-1 y 1,93 - 7,95 kg de lechevaca-1 día-1, cuando son utilizados en el menor y en el mayor, nivel de consumo, respectivamente.

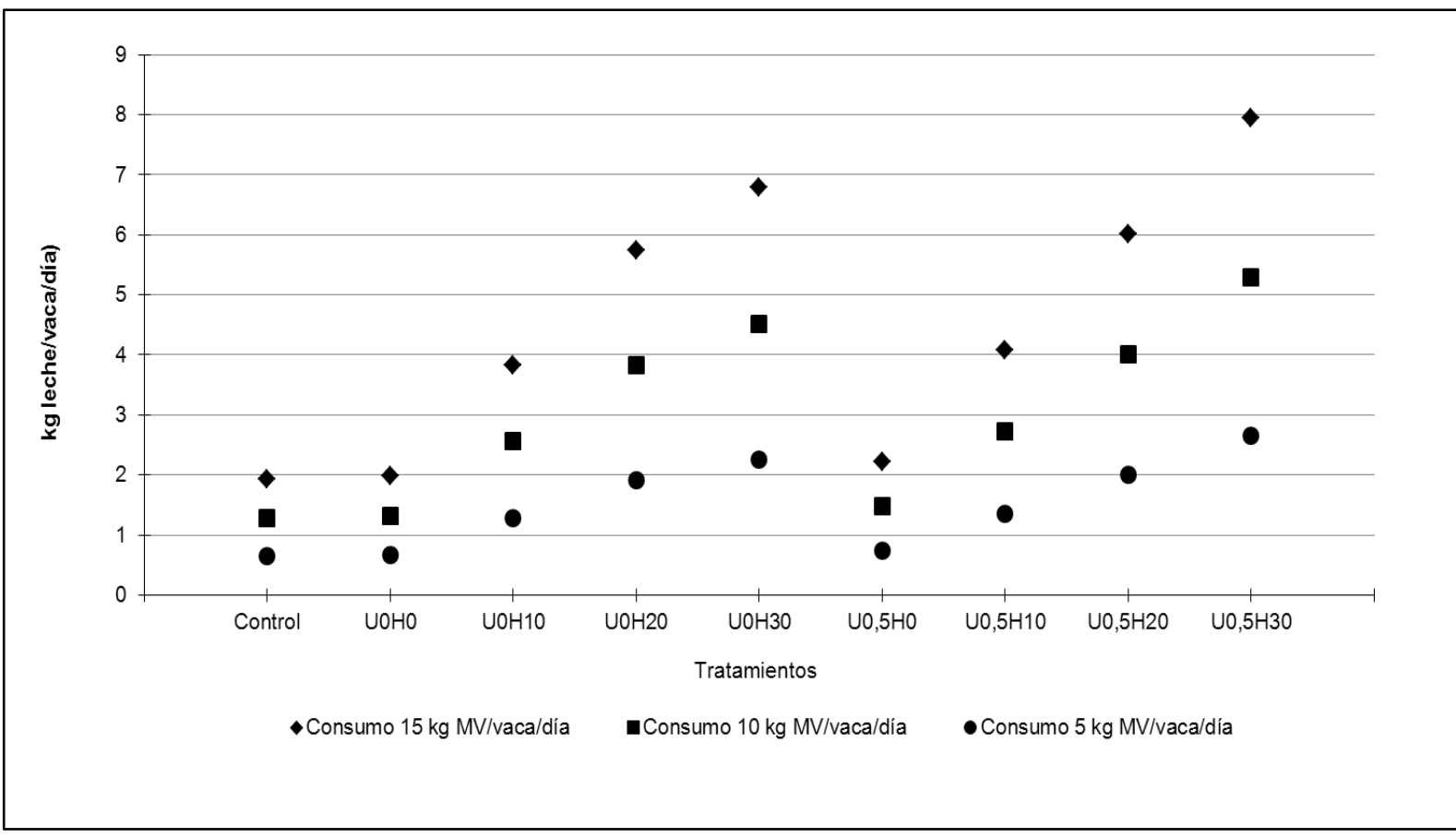

Figura 2. Potencial de producción por $E N_{L}$, de los ensilados de corona de piña con pulpa de cítricos deshidratada, heno y urea de acuerdo al nivel de consumo de Materia verde (MV) (5, 10 y $15 \mathrm{~kg} \mathrm{MV/día).} \mathrm{H=(Heno)} \mathrm{y} \mathrm{U=(Urea)}$ 


\section{CONSIDERACIONES FINALES}

El uso de heno como aditivo para ensilajes permite el aumento en el contenido de MS, situación que favorece el proceso de conservación del forraje, pero impacta de manera negativa la calidad nutricional de las coronas de piña, ya que aumenta la concentración de fibra, lo que podría reducir el consumo y la digestibilidad de los materiales ensilados.

La adición de urea permite mejorar el contenido de proteína cruda, debido a un aumento en la concentración de nitrógeno no proteico, sin embargo puede afectar el proceso de ensilaje, por un aumento en la capacidad amortiguadora, lo que se refleja en valores mayores de $\mathrm{pH}$ y NH3/NT, tal y como se observó en este trabajo, situación que obliga a tomar medidas para asegurar una adecuada disminución del $\mathrm{pH}$; tal como aumentar la concentración de azúcares solubles, con la finalidad de mantener la calidad del material a conservar.

La degradabilidad de la PC de los tratamientos fluctuó entre 71 y $88 \% \mathrm{~h}^{-1}$, esto se debe a un aumento en la concentración de la PC, ocasionado por la inclusión de urea, lo que indica un enriquecimiento del material, que podría mejorar la productividad del animal.

Se realizaron simulaciones para determinar el potencial productivo de los materiales ensilados de acuerdo al consumo y aporte de PC y de $E N_{L}$, se determinó que los tratamientos ensilados podrían producir entre $0,34-7,65 \mathrm{~kg}_{\text {leche }}$ vaca $^{-1}$ día $^{-1}$, de acuerdo al nivel de consumo (5, 10 y $15 \mathrm{~kg} \mathrm{MV/día),} \mathrm{cuando} \mathrm{son} \mathrm{utilizados} \mathrm{como} \mathrm{complemento} \mathrm{de}$ una dieta balanceada. 


\section{LITERATURA CONSULTADA}

ARBABI S., GHOORCHI T., NASERIAN A.A. 2008. The effect of dried citrus pulp, dried beet sugar pulp and wheat straw as silage additives on by-products of orange silage. Asian Journal of Animal Science 2(2) 35-42.

ARROYO C., ROJAS-BOURRILLON A., ROSALES R. 2003. Uso de urea o pollinaza como suplemento proteico para toretes consumiendo ensilaje de pulpa de pejibaye. Agronomía Costarricense 27(2): 69 - 73.

ASSOCIATION OF OFFICIAL ANALYTICAL CHEMIST (AOAC). 1998. Official Methods of Analysis of AOAC International. 16th ed, 4th rev. Gaithersburg, MD: AOAC International.

AZEVÊDO J.A., FILHO S.C., DETMANN E., PINA D., PEREIRA L.G., DE OLIVEIRA K., FERNANDES H., SOUZA N.K. 2011. Predição de fraçõesdigestíveis e valor energético de subprodutos agrícolas e agroindustriais para bovinos. Revista Brasileira de Zootecnia 40(2): 391 - 402.

BATHOLOMEW D.P., PAULL R.E., ROHRBACH K.G. 2003. Pineapple: Botany and uses. CABI Publishing. United Kingdom. $313 p$.

BELYEA R., STEEVENS B., GARNER G., WHITTIER J., SEWELL H. 1996. Using NDF and ADF To Balance Diets. MissouryUniversityExtension: G3161. USA.

BETANCOURT J.C, 2004. Caracterización nutricional y productiva de material fresco y ensilado de maní forrajero (Arachis pintoi) cultivado en asocio con maíz (Zea mays), a tres densidades de siembra. Tesis de Maestría en Ciencias Agrícolas y Recursos Naturales. Universidad de Costa Rica. Costa Rica. 110 pp.

BETANCOURT M., GONZALEZ I., MARTINEZ DE ACURERO M. 2005. Evaluación de la calidad de los ensilajes. Revista Digital Centro Nacional de Investigaciones Agropecuarias de Venezuela. No 8 mayo-agosto. Maracay, Aragua, Venezuela. 1$5 p$ 
BORGES J.A., BASTARDO Y., SANDOVAL E., BARRIOS M., ORTEGA R. 2011. Efecto de la adición de urea y el tipo de fermentación en la estabilidad de silajes de Caña de Azúcar (Saccharumspp.). Zootecnia Tropical 29(3): 283-291.

BOSCHINI-FIGUEROA C., PINEDA-CORDERO L., CHACÓN-HERNÁNDEZ P. 2014. Evaluación del ensilaje del pasto Ratana (IschaemumindicumHoutt.) con tres diferentes aditivos. Agronomía Mesoamericana 25(2), 297-311.

CASTILLO M., ROJAS-BOURRILLÓN A., WINGCHING-JONES R. 2009. Valor nutricional del ensilaje de maíz cultivado en asocio con vigna (Vigna radiata). Agronomía Costarricense 33(1): $133-146$.

CHACÓN-HERNÁNDEZ P.A., VARGAS-RODRÍGUEZ C.F. 2009. Digestibilidad y calidad del Pennisetum purpureum cv. King grass a tres edades de rebrote. Agronomía Mesoamericana 20(2): $399-408$.

CORREIA M.X., COSTA R.G., DA SILVA J.H., DE CARVALHO F.F., DE MEDEIROS A. 2006. Utilização de resíduo agroindustrial de abacaxidesidratadoem dietas para caprinos emcrescimento: digestibilidade e desempenho. Revista. Brasileira de Zootecnia 35(4) 1822-1828 (supl.)

CUBERO J.F., ROJAS A., WINGCHING R. 2010. Uso del inóculo microbial elaborado en finca en ensilaje de maíz (Zea mays). Valor nutricional y fermentativo. Agronomía Costarricense 34(2): 237-250.

CUNHA M.D.G., OLIVEIRA E., RAMOS J.L., ALCÂNTARA M.D. 2009. Conservação e utilização do resíduo de abacaxi na alimentação de ovinos no curimataúocidental da paraíba. Revista Tecnologia\&Ciência Agropecuaria 3 (3): 55-62.

DETMANN E., VALHADARES FILHO S.C. 2010. Sobre a estimação de carboidratosnão fibrosos em alimentos e dietas. Arquivo Brasileiro de Medicina Veterinária e Zootecnia. 62(4):980 - 984.

DI RIENZO J A., CASANOVES F., BALZARINI M.G., GONZALEZ L., TABLADA M., ROBLEDO Y.C. 2013. InfoStat versión 2013. Grupo InfoStat, FCA, Universidad Nacional de Córdoba, Argentina. URL http://www.infostat. com. ar. 
EASTRIDGE M. 2002. Dairy NRC has improvements in energy calculations. Feedstuffs Newspaper.Nutrition and Health. July 8th. 11-13.

ELIZONDO-SALAZAR J.A., CAMPOS-GRANADOS C.M. 2014.Características nutricionales de la cáscara de piña ensilada con cantidades crecientes de urea y heno. Nutrición Animal Tropical 8(2): 51- 71.

GUTIÉRREZ F., ROJAS A., DORMOND H., POORE M., WINGCHING R. 2003. Características nutricionales y fermentativas de mezclas ensiladas de desechos de piña y avícolas. Agronomía Costarricense. 27(1): 79 - 89.

HIRIART M. 2008. Ensilados. Procesamiento y Calidad. Editorial Trillas. México. 110p.

JONES, R., JONES, D.I.H. 1996. The effect of in-silo effluent absorbents on effluent production and silage quality. Journal of Agricultural Engineering Research 64(3): 173-186.

KELLEMS R.O., WAYMAN O., NGUYEN A.H., NOLAN J.C., CAMPBELL C.M., CARPENTER J.R., HO-A E.B. 1979. Post-harvest pineapple plant forage as a potential Feedstuff for beef cattle: evaluated by laboratory Analyses, $/ \mathrm{n}$ vitro and/n v/vodigestibility and Feedlot trials. Journal of Animal Science 48 (5): 1040-1048.

LALLO F.H., NUNES I., GONÇALVES W., ZEOULA L.M., BARROS F., YOSHIMI F. 2003. Níveis de substitução da silagem de milho pela silagem de residuos industriais de abacaxi sobre a degradabilidade ruminal em bovinos de corte. Revista Brasileira de Zootecnia 32(3): $719-726$.

LÓPEZ M., WINGCHING R., ROJAS A. 2009. Características fermentativas y nutricionales del ensilaje de rastrojo de piña (Ananas comosus). Agronomía Costarricense 33(1): $1-15$.

LÓPEZ M., WINGCHING R., ROJAS A., RODRÍGUEZ S. 2014a. Valor nutricional del ensilaje de rastrojo de piña con niveles de crecientes de urea. Nutrición Animal Tropical 8(1): $1-20$. 
LÓPEZ M., WINGCHING R., ROJAS A. 2014b. Meta-análisis de los subproductos de piña (Ananascomosus) para la alimentación animal. Agronomía Mesoamericana 25(2): 383-392.

LOUSADA JR. J.E, MIRANDA J., RODRÍGUEZ N., MACHADO J.C., BRAGA R. 2005. Consumo e digestibilidade de subprodutos do processamento de frutas em ovinos. RevistaBrasileira de Zootecnia 34 (2): 659-669.

MCDONALD P. 1981. The biochemistry of silage.John Wiley Ltd. New York. 226 p.

MCDONALD P., HENDERSON A.R. 1962. Buffering capacities of herbage samples as factor of silage. Journal of Science Food and Agriculture 13:395-400.

MERTENS D. 1996. Formulating Dairy Rations. Using fiber and Carbohydrate Analyses to formulate dairy rations.U.S Dairy Forage Research Center.Informational Conference with dairy and forage industries. $81-92$.

MOORE K.J., PETERSON M.A. 1995. Post-harvestphysiology and preservation of forages. Crop Science Society of America Inc. Special publication N. 22. Wisconsin, USA. 91-107 p.

MORENO A. H. 1977. Evaluación de ensilajes de pasto Panamá (Saccharum sinense), para la alimentación de vacas de doble propósito. Tesis de maestría, Universidad de Costa Rica-Centro Agronómico Tropical de Investigación y Enseñanza, Turrialba, Costa Rica. 98 p.

NATIONAL RESEARCH COUNCIL (NRC). 2001. Nutrient Requirements of Dairy Cattle. 7th ed. National Academy Press. Washington DC. 408 p.

NEUMANN M., OLIBONI R., OLIVEIRA M.R., FARIA M.V., UENO R.K., REINERH L.L., DURMAN T. 2011.Chemicals additive used in silages. Applied Research \& Agrotechnology 3(2): 187-208.

NHAN N.T.H., HON N.V., PRESTON T.R. 2009. Ensiling with or without additives to preserve pineapple residue and reduce pollution of the environment. Livestock Research for Rural Development 21(7). Retrieved March 8, 2012, at 10:15 am, from http://www.Irrd.org//rrd21/7/nhan21096.htm 
PEÑA P. M., DEL POZO P. 1992. Explotación de pastos y forrajes. ISCAH: La Habana, Cuba. $106 \mathrm{p}$.

PEREIRA E.S., FILHO J.G.; FREITAS E.R., NEIVA J.N., CÂNDIDO M.J. 2009. Valor energético de subprodutos da agroindústria brasileira. Archivos de Zootecnia 58(223): $455-458$.

POND W. G., CHURCH D. C., POND K. R. 2003.Fundamentos de nutrición y alimentación de animales. LIMUSA WILEY. México D.F. 636 p.

PRADO I., LALLO F.H., ZEOULA L.M., NETO S., GONÇALVES W., MARQUES J. 2003. Níveis de substitução da silagem de milho pela silagem de resíduosindustriais de abacaxi sobre el desempenho de bovinos confinados. Revista Brasileira de Zootecnia 32(3): 737 - 744.

RAZAK O.A., MASAAKI H., YIMAMU A., MEIJI O. 2012. Potential Water Retention Capacity as a Factor in Silage Effluent Control: Experiments with High Moisture Byproduct Feedstuffs. Asian-Australasian Journal of Animal Sciences 25(4): 471-478.

Rebolledo A., PÉReZ A.L., REBolledo L., BeCerRiL A.E. 2006. Rendimiento y calidad de fruto de cultivares de piña en densidades de plantación. Revista Fitotecnia Mexicana 29(1): 55 - 62.

RODRÍGUEZ S., LÓPEZ M., WINGCHING-JONES R., Rojas A. 2014. Adición de melaza deshidratada y urea en ensilados de rastrojos de piña. Agronomía Mesoamericana 25(2):313-321.

ROGÉRIO M.C.P., BORGES I., NEIVA J.N.M., RODRIGUEZ N.M., PIMENTEL J.C.M., MARTINS G.A., RIBEIRO T. P., COSTA J.B., SANTOS S.F., CARVALHO F.C. 2007. Valor nutritivo do resíduo da industria processadora de abacaxi (ananas comosus l.) em dietas para ovinos. 1. Consumo, digestibilidade aparente e balanços energético e nitrogenado Arquivo Brasileiro de Medicina Veterinária e Zootecnia 59(3):773 - 781. 
SÁNCHEZ J. 2010. Práctica en el Programa de Transferencia Tecnológica de la Cooperativa de Productores de Leche Dos Pinos R.L. Ciudad Quesada, San Carlos. Práctica de Bachillerato. Escuela de Zootecnia, Universidad de Costa Rica, Costa Rica 31 p.

SÁNCHEZ J. M., SOTO H. 1998. Estimación de la calidad nutricional de los forrajes del cantón de San Carlos. II. Componentes de la pared celular. Revista Nutrición Animal Tropical (Costa Rica) 4(1):7-19.

SÁNCHEZ J. M., SOTO H. 1999. Estimación de la calidad nutricional de los forrajes del cantón de San Carlos. III. Energía para la producción de leche. Revista Nutrición Animal Tropical (Costa Rica) 5(1):31-49.

TOBÍA C. 2004. Introducción del ensilaje de soya en un sistema de producción intensiva de leche en el trópico húmedo de Costa Rica. Tesis de doctorado, Universidad de Costa Rica, San José, Costa Rica. 120 p.

VAN SOEST P.J., ROBERTSON, J.B. 1985. Analysis of forages and fibrous food.AS 613. Cornell University, A Laboratory Manual. Deparment of Animal Science. Ithaca NY. $613 \mathrm{p}$.

VAN SOEST P.J., ROBERTSON, J.B., LEWIS B.A. 1991. Methods for dietary fiber, neutral detergent fiber, and non starch polysaccharides in relation to animal nutrition. Journal of Dairy Science 74:3583-3597

VILLALOBOS L., ARCE J. 2014. Evaluación agronómica y nutricional del pasto estrella africana (Cynodon nlemfuensis) en la zona de Monteverde, Puntarenas, Costa Rica. II. Valor nutricional. Agronomía Costarricense 38(1): 133 - 145.

WEISS W.P. 2004.Fine-tuning energy calculations. In: Eastridge M. Proceedings of the Tri-State Dairy Nutrition Conference. Purdue University, Michigan State University, Ohio State University. April 27 - 28. Fort Wayne, Indiana, United States. 131 142.

WEISS W.P., FISHER G.R., ERICKSON G.M. 1989. Effect of source of neutral detergent fiber and starch on nutrient utilization by dairy cows. Journal of Dairy Science 72(9): $2308-2315$. 
WINGCHING R., ALVARADO G. 2009. Valor nutricional del heno de Transvala inoculado con el hongo Pleurotusostreatussp. Agronomía Costarricense. 33(1): 147-153.

YAN T., AGNEW R.E. 2004. Prediction of nutritive values in grass silages: II. Degradability of nitrogen and dry matter using digestibility, chemical composition and fermentation data. Journal of Animal Science 82: 1367 - 1379.

YITBAREK M.B., TAMIR B. 2014. Silage Additives: Review. Open Journal of Applied Sciences 4: 258-274. 